\title{
A Novel Tumor Suppressor Gene, TMEM171, Inhibits the Development of Gastric Cancer by Dephosphorylating IKBa and Inactivating the NF-kB Signaling Pathway
}

\section{De-feng Li}

Shenzhen People's Hospital https://orcid.org/0000-0003-3118-6840

Qi Tang

Shenzhen People's Hospital

Mei-feng Yang

Yantian District people hospital

Yu-ling Bing

zhuzhou central hospital

Quan-zhou Peng

Shenzhen People's Hospital

Li-sheng Wang ( $\nabla$ wanglsszrmyy@163.com )

Shenzhen People's Hospital

Jun Yao

Shenzhen People's Hospital

\section{Research Article}

Keywords: Gastric cancer, TMEM171, IKBa/ NF-kB signaling pathway

Posted Date: February 24th, 2022

DOI: https://doi.org/10.21203/rs.3.rs-1361540/v1

License: (c) (i) This work is licensed under a Creative Commons Attribution 4.0 International License. Read Full License 


\section{Abstract}

Background: Gastric cancer (GC) is one of the most common malignant tumors with an unsatisfactory prognosis worldwide. Therefore, it is urgently necessary to explore the mechanism underlying the tumorigenesis of GC.

Methods: In the present study, we identified that TMEM171 was down-regulated in GC tissues, and its expression was closely associated with the prognosis of GC patients based on the data from Gene Expression Omnibus (GEO) and the Cancer Genome Atlas (TCGA). Subsequently, qRT-PCR, Western blotting analysis, and immunohistochemistry (IHC) assay were performed to detect the expression of TMEM171 in GC tissues and cell lines. The functional roles and underlying mechanisms of TMEM171 involved in GC growth were also explored in vitro and in vivo.

Results: TMEM171 was remarkably down-regulated in GC tissues and GC cell lines. Functionally, overexpression of TMEM171 suppressed the proliferation of GC cells and promoted the apoptosis of GC cells, whereas depletion of TMEM171 contributed to the opposite trend in vitro and in vivo. TMEM171 functioned as an inhibitor for GC proliferation through dephosphorylating $\mathrm{I} \mathrm{KB}$ and then inactivating the canonical NF-KB signaling pathway.

Conclusions: This study revealed the vital role of TMEM171 in GC, highlighting the regulatory function of the TMEM171/lkBa/NF-kB signaling pathway in modulating GC progression. Collectively, our findings provided a novel prognostic and therapeutic target for GC patients.

\section{Introduction}

As one of the most common malignancies, gastric cancer (GC) has 19.3 million new cases and 10 million cancer-related deaths in $2020^{1}$. GC ranks as the fifth most frequently diagnosed cancer, and it is the third leading cause of cancer-related deaths worldwide ${ }^{1}$. Despite recent advances in therapeutic modalities including surgical resection, adjuvant chemotherapy and radiotherapy, most GC patients are diagnosed at an advanced stage with poor prognosis ${ }^{2}$. Unfortunately, there is asymptomatic manifestation at the early disease stage of the disease, which contributes to delayed diagnosis and missed opportunities for effective treatments ${ }^{3}$. Therefore, it is vital to improve the early-stage diagnosis rate of GC patients and subsequently increase the long-term survival of GC patients.

To date, the molecular mechanism underlying the tumorigenesis of GC remains unclear. A variety of risk factors are involved in the occurrence and development of GC, such as environmental factors and molecular aberrations. Environmental risk factors include smoking, alcohol, Helicobacter pylori (H. pylori) infection and so on ${ }^{4,5}$. Molecular aberrations mainly include the dysregulation of oncogene and tumorsuppressor genes ${ }^{6,7}$. Due to the development of high-throughput sequencing, several cancer-related genes, including both coding genes and non-coding genes are identified in $\mathrm{GC}^{6-10}$. Despite developing 
targeted therapy of these dysregulated genes, the prognosis of GC still remains unsatisfactory. Therefore, it is urgently necessary to explore the novel molecular mechanism and develop novel targets.

In the present study, we found that TMEM171, a novel tumor-suppressor gene, was down-regulated in GC tissues using bioinformatic analysis from Gene Expression Omnibus (GEO). Moreover, we found that GC patients with low expression of TMEM171 had poorer overall survival (OS) compared with its high expression group based on data from GEO and The Cancer Genome Atlas (TCGA). Subsequently, we demonstrated that overexpression of TMEM171 suppressed proliferation of GC cells and enhanced the apoptosis of GC cells, whereas depletion of TMEM177 expression suppressed the apoptosis of GC cells and enhanced the proliferation of GC cells in vitro. In addition, further experiments indicated that TMEM171 inhibited GC growth in vivo. Importantly, we verified that TMEM171 could inhibit IKBa phosphorylation, and then inactivate the NF-kB signaling pathway both in vivo and in vitro. Therefore, TMEM171 exerted its functions via regulating the NF-kB signaling pathway. These findings revealed that TMEM171 might act as a promising prognostic marker and therapeutic target for GC patients.

\section{Methods}

\section{Data source}

In the GEO (https://www.ncbi.nlm.nih.gov/geo/) repository, the microarray data of GSE51575 (containing 26 GC samples and 26 normal samples), GSE118916 (containing 15 GC samples and 15 normal samples), GSE79973 (containing $10 \mathrm{GC}$ samples and 10 normal samples), GSE81948 (containing 15 GC samples and 5 normal samples) and their clinical data were downloaded. The RNA-seq FPKM (reads per kilobase per million) data of $375 \mathrm{GC}$ samples and 32 normal samples, as well as corresponding clinical characteristics were downloaded from TCGA (https://portal.gdc.cancer.gov). The GSE62254 (including 266 GC patients), GSE84437 (including 431 GC patients), GSE26253 (including 432 GC patients), and their clinical characteristics were downloaded as the validation set after the unavailable survival data were excluded.

\section{Data analysis}

The differentially expressed genes (DEGs) were calculated by comparing the GC samples with normal samples using the limma package in R 4.0 software based on the criteria of $\mid \log 2$ fold change $(\mathrm{FC}) \mid \geq 1$ and false discovery rate (FDR) $<0.05$ from the GSE51575, GSE118916, GSE79973 and GSE81948 datasets.

Overlapping DEGs were obtained using the VennDiagram package based on the DEGs of the GSE51575, GSE118916, GSE79973, and GSE81948 datasets. Univariate Cox regression analyses were performed to identify prognosis-associated DEGs from these overlapping DEGs by the survival package in R software based on the GSE62254 dataset.

The least absolute shrinkage and selection operator (LASSO) Cox model was constructed for the selection of key prognosis-associated DEGs using the GImnet package. Subsequently, a risk score model was constructed based on the expression quantity and coefficients of prognosis-associated DEGs. The 
risk score was calculated for each GC patient as follows: Risk scores $=\beta 1^{\star} E x p 1+\beta{ }^{\star} E x p 2+\beta i^{\star} E x p i$, where $\beta$ represented the coefficient score, Exp represented the gene expression, and $i$ represented the $i^{\text {th }}$ DEG.

To validate the risk score model, the GC patients were divided into low-risk and high-risk group according to the median risk score, and a log-rank test was conducted to assess the survival difference between the low-risk group and high-risk group using the survival package in R software based on GSE62254, GSE84437, and GSE26253. Moreover, receiver operating characteristics (ROC) curves were drawn to determine the predictive capability of the prognostic model using the survivalROC package in $\mathrm{R}$ software.

The differential expressions of the prognosis-associated DEGs between GC samples and normal samples were calculated based on the GSE51575, GSE118916, GSE79973 and GSE81948 datasets. Progressionfree survival (PFS) of each prognosis-associated DEG was determined using survival and survminer packages based on the GSE26253 dataset. Pearson correlation analysis was performed to identify the genes associated with the prognosis-associated DEGs using BiocManager packages, and enrichment pathway analysis was used to determine the biological functions of the prognosis-associated DEGs through clusterProfiler, org.Hs.eg.db, enrichplot and ggplot2 packages based on the GSE62254 dataset.

\section{Cell lines and culturing}

GES-1 cells were obtained from the Cancer Institute and Hospital of the Chinese Academy of Medical Sciences (Beijing, China). HCG-17 cells were purchased from the Type Culture Collection of the Chinese Academy of Science (Shanghai, China). NCl-N87 and Hs-746T cells were provided by the American Type Culture Collection (Manassas, USA). MKN-7 cells were supplied by the Japanese Collection of Research Bioresources (Tokyo, Japan). GSE-1 and NCI-N87 cell lines were maintained in Dulbecco's Modified Eagle Medium (DMEM) (Procell, Wuhan, China) supplemented with 10\% fetal bovine serum (FBS) (Gibco, NY, USA) and $1 \%$ penicillin-streptomycin (NCM Biotech, Suzhou, China) at $37^{\circ} \mathrm{C}$ in a humidified atmosphere containing 5\% CO2. HCG-27, Hs-746T, and MKN-7 were maintained in RPMI-1640 medium (Procell, Wuhan, China) supplemented with $10 \%$ FBS and $1 \%$ penicillin-streptomycin at $37^{\circ} \mathrm{C}$ in a humidified atmosphere containing $5 \% \mathrm{CO}_{2}$.

\section{Plasmids and siRNA transfection and lentiviral transduction}

The full-length sequence of TMEM171 (Tsingke, Shanghai, China) was cloned to construct the TMEM171 overexpression vector. siRNAs targeting TMEM171 were designed and synthesized by Tsingke (Shanghai, China). The lentiviral vectors (pGLV3/GFP/Puro) containing the overexpression vector or shRNAs targeting TMEM171 were generated, which were transduced into cell lines (The sequences were available in Table S1.).

\section{Cell proliferation assay}

Cell proliferation assays were performed using CCK-8 reagents (Sigma, New Jersey, USA) according to the manufacturer's instructions. 


\section{Cell apoptosis assay}

The number of apoptotic cells was evaluated using the Annexin V-FITC/Propidium lodide (PI) Apoptosis Detection Kit (KeyGen Biotech, Nanjing, China) according to the manufacturer's instructions. The cells were analyzed using flow cytometry, and all apoptosis data were calculated using Flowjo software (Ashland, Kentucky, USA).

\section{Flow cytometry}

For cell cycle experiments, the cells were stained with PI (Beyotime, Shanghai, China) according to the manufacturer's instructions. The cells were analyzed using flow cytometry, and the proportions of different types of cells in the G0/G1, S and G2/M phases were calculated (Ashland, Kentucky, USA).

\section{RNA extraction and qRT-PCR}

RNA extraction and qRT-PCR were carried out as previously described ${ }^{11,12}$. Primer sequences used in the present study were available in Table S1.

\section{Protein extraction and Western blotting analysis}

Protein extraction and Western blotting analysis were conducted as previously described ${ }^{11}{ }^{13}$. Antibodies against TMEM171 were purchased from Thermo Fisher (NY, USA). Antibodies against IKBa, and p-IKBa were obtained from Abcam (Cambridge, UN). Antibodies against PCNA, MCM2 and GAPDH were supplied by Proteintech (Rosemont, USA). Goat anti-rabbit IgG was provided by Cell Signaling Technology (Boston, USA). The diluted antibodies of TMEM171 (1: 1,000), IKBa (1:2,000), p-IKBa (1:1,000), PCNA $(1: 4,000)$, MCM2 $(1: 2,000)$, and GAPDH $(1: 6,000)$, as well as goat anti-rabbit IgG $(1: 10,000)$, were used in Western blotting analysis.

\section{Immunohistochemistry (ICH)}

IHC assay was carried out as previously described ${ }^{11,14}$. Antibodies against PCNA and MCM2 were supplied by Proteintech (Rosemont, USA). The diluted antibodies of PCNA (1:500) and MCM2 (1:100), as well as goat anti-rabbit $\lg G$ (1:250), were used in the ICH assay.

\section{Xenograft mouse model}

All male BALB/c nude mice (5-6 weeks old) were purchased from Hunan SJA Laboratory Animal Co (Changsha, China). A total of 12 mice were divided into two groups. HCG-27 cells were stably transfected with TMEM171 overexpression and NC vectors using lentivirus. pLv-control or TMEM171-expressing HCG27 cells $\left(5 \times 10^{6}\right)$ were subcutaneously injected into the axillary region of nude mice. After 8 days, the tumor volume was measured using the following formula: $\mathrm{V}\left(\mathrm{cm}^{3}\right)=0.5 \times($ length $) \times(\text { width })^{2}$ once every 5 days. The mice were sacrificed 4 weeks after the injection. The xenograft tumors were harvested and stored in liquid nitrogen for further analysis. 


\section{Statistical analysis}

Data were presented as the mean \pm SD. A two-tailed Student's t-test was used to assess the differences between the two groups. Comparisons among three groups were analyzed using a one-way analysis of variance (ANOVA) following Bonferroni's post-hoc test. The chi-squared test was applied to evaluate the association between the expression of EMTM171 and clinicopathological characteristics. Survival analysis was performed using the Kaplan-Meier analysis with the log-rank test. A P-value of $<0.05$ was considered statistically significant. All statistical analyses were carried out using SPSS software 17.0 (SPSS Inc., USA) and GraphPad Prism 7.0 (GraphPad Software, USA).

\section{Results}

\section{Identification of DEGs and bioinformatic analysis.}

The GEO was utilized to obtain the differentially expressed genes (DEGs) from GC samples compared with normal tissues, and Fig. 1A-D show the obtained DEGs ( $|\log F C|>1$, adj. $P$ value $<0.05)$. Figure $1 \mathrm{E}$ illustrates the Venn diagram of the DEGs in these four microarray datasets (GSE51575, GSE118916, GSE79973, and GSE81948). Finally, 245 DEGs, including 164 up-regulated and 81 down-regulated DEGs, were obtained.

Univariable Cox regression was performed to determine the prognosis-associated DEGs from these 245 candidate DEGs in GC based on the GSE62254 dataset. As table S2 shown, 30 DEGs associated with overall survival (OS) were obtained. Furthermore, 13 key prognosis-associated DEGs (TGFBI, GPSM2, STIL, LIFR, CD36, COL4A1, CYFIP2, CKAP2, FAM83D, GPR155, ANKRD22, TMEM171 and STX19) were identified using the LASSO analysis (Fig. 2A-B). Therefore, a risk score model was constructed based on these 13 prognosis-associated DEGs. The risk score of each GC patient was calculated as follows: Risk scores $=(-2.846)$ * expression quantity of TGFBI +12.050 * expression quantity of GPSM $2+6.432$ * expression quantity of STIL $+(-4.201)$ * expression quantity of LIFR $+(-0.499)$ * expression quantity of $\mathrm{CD} 36+(-9.886)$ * expression quantity of COL4A1 + $(-4.155)$ * expression quantity of CYFIP2 + 8.356 * expression quantity of CKAP2 + (-17.812) * expression quantity of FAM83D + $(-2.611)$ * expression quantity of GPR155 + 1.232 * expression quantity of ANKRD22 + 2.724 * expression quantity of TMEM171 +16.211 * expression quantity of STX19.

Survival analysis was performed to assess the predictive performance of the risk score model based on the GSE62254, GSE84437, and GSE26253 datasets. Subsequently, GC patients were divided into the lowrisk and high-risk groups based on the median risk score. The results revealed that the patients with higher risk score had a poorer OS compared with the low-risk group. Meanwhile, the distribution of the risk score curve and the survival status were ranked by the risk score, and showing the high-risk patient had a relatively poor clinical outcome (Fig. 2C-E). Furthermore, ROC analysis revealed that the risk score model had a satisfactory sensitivity and specificity of predictive prognosis values for GC patients at 1-, 2-, 3-, 4-, and 5- years (Fig. 2F-2H). 
We examined the expressions of the 13 key prognosis-associated DEGs (TGFBI, GPSM2, STIL, LIFR, CD36, COL4A1, CYFIP2, CKAP2, FAM83D, GPR155, ANKRD22, TMEM171, and STX19) between GC samples and normal samples based on the GSE51575, GSE118916, GSE79973, and GSE81948 datasets. The results showed that TGFBI, STIL, GPSM2, FAM83D, COL4A1 and CKAP2 were up-regulated in GC samples, whereas LIFR, CD36, CYFIP2, GPR155, ANKRD22, TMEM171 and STX19 were down-regulated in GC samples (Figure S1A-S1D). Subsequently, progression-free survival (PFS) analysis of the 13 key prognosis-associated DEGs was performed based on the GSE26253 dataset, showing that TMEM171 and TGFBI were closely associated with PFS (Figure S2). It has been reported that TGFBI is closely associated with the occurrence and development of $\mathrm{GC}^{15}$. However, the association between TMEM171 and the pathogenesis of GC remains unclear.

Subsequently, the survival analysis revealed that the GC patients with a higher expression of TMEM171 had a better OS compared with its low-expression group based on the TCGA dataset (Figure S3A). Moreover, TMEM171 had a satisfactory sensitivity and specificity of predictive prognosis values for GC patients at 1- and 3- years using ROC analysis (Figure S3B). Furthermore, multivariate regression analysis revealed that TMEM171 was closely associated with the prognosis of GC patients (Figure S3C) based on the GSE62254 dataset. Functional enrichment analysis showed that TMEM171 was mainly correlated with the ІкВа/NF-кB signaling pathway, tight junction, and actin cytoskeleton organization (Figure S3D).

\section{TMEM171 is down-regulated in GC}

To further verify the expression of TMEM171 in GC tissues, we performed qRT-PCR on GC tissues and noncancerous control tissues $(n=20)$. The results revealed that the expression of TMEM171 at the mRNA level in GC tissues was significantly down-regulated compared with the noncancerous control tissues (Fig. 3A). Moreover, the immunohistochemistry (IHC) assay showed that the expression of TMEM171 at the protein level was significantly decreased in GC tissues (Fig. 3B). Subsequently, the expression of TMEM171 was examined in GC cell lines (NCI-N87, MKN7, Hs-746T, and HGC-27) and human normal gastric epithelial cell line (GES-1) by qRT-PCR and Western blotting analysis. These results revealed that the expression of TMEM171 in NCI-N87, MKN7 and HGC-27 cells was significantly down-regulated relative to that in GES-1 cells, whereas the expression of TMEM171 was significantly up-regulated in Hs746T cells compared with GES-1 cells (Fig. 3C-D). Among these GC cell lines, HGC-27 cells had the lowest expression of TMEM171. Therefore, we selected Hs-746T and HGC-27 cells to further explore the underlying mechanism of TMEM171 in the tumorigenesis of GC.

\section{TMEM171 inhibits the proliferation of GC cells in vitro}

To examine the function of TMEM171 in GC, we established TMEM171-overexpressing HGC-27 cells and TMEM171-depleted Hs-746s (Figure S4A-4D). Depletion of TMEM171 significantly promoted the proliferation of GC cells, inhibited the apoptosis of GC cells, and decreased the proportion of cells in the G1 phase (Fig. 4A-4E). However, overexpression of TMEM171 contributed to an opposite trend, showing suppressed the proliferation of GC cells, promoted the apoptosis of GC cells, and an increased the 
proportion of cells in the G1 phase (Fig. 4F-4I). Moreover, depletion of TMEM171 enhanced the expressions of MCM2 and PCNA at the protein level, whereas overexpression of TMEM171 significantly inhibited the expressions of MCM2 and PCNA at the protein level (Fig. 4J-4K).

\section{TMEM171 inhibits proliferation of GC cells in vivo}

To explore the effect of TMEM171 on the regulation of tumor growth in vivo, HGC-27 cells stably overexpressing TMEM171 were subcutaneously injected into nude mice (Fig. 5A). We found that the growth of xenografts with high expression of TMEM171 was slower, and the tumor size was smaller compared with the control xenografts with low expression of TMEM171 (Fig. 5A-5B). The expression of TMEM171 at the protein level was significantly higher in the TMEM171-high group compared with the TMEM171-low group (Fig. 5C). IHC staining in tumor specimens revealed that the expressions of MCM2 and PCNA at the protein level were lower in the TMEM171-high group compared with the TMEM171-low group (Fig. 5D). These results suggested that TMEM171 could inhibit the growth of GC cells.

\section{TMEM171 inactivates the NF-KB signaling pathway}

It has been reported that the NF-KB signaling pathway plays a crucial role in the tumorigenesis of human cancer, and the genes targeting this pathway can be applied as promising markers and chemotherapeutic targets in clinical practice ${ }^{16,17}$. In addition, IKBa plays an almost exclusive role as an inhibitor of the NF$\mathrm{KB}$ signaling pathway ${ }^{18}$. The primary mechanism underlying the activation of canonical NF-KB signaling pathway is the degradation of $\mathrm{IKBa}$, and the inhibitor of NF-KB kinase (IKK) that phosphorylates $1 \mathrm{KBa}$ and leads to its ubiquitination and degradation plays a critical role ${ }^{19}$. In the present study, the bioinformatic analysis revealed that TMEM171 regulated the $\mathrm{IKBa} / \mathrm{NF}-\mathrm{KB}$ signaling pathway (Figure S2D). Therefore, to determine whether TMEM171 regulated the NF-KB signaling pathway via IKBa, we detected the expression and phosphorylation status of IKBa in GC cells following TMEM171 overexpression or depletion. The results showed that the overexpression or depletion of TMEM171 exhibited no significant effect on the expression of IKBa at the protein level (Fig. 6A-6B). However, overexpression of TMEM171 contributed to reduced protein phosphorylation of ІкBa, while depletion of TMEM171 resulted in increased protein phosphorylation of IKBa (Fig. 6A-6B). Moreover, in mouse xenografts, overexpression of TMEM171 also led to reduced protein phosphorylation of IKBa (Fig. 6C). Indeed, an NF-KB signaling pathway inhibitor, PDTC, could restore the low expression of TMEM171 in TMEM171-depleted Hs-746T cells, promote cell proliferation, inhibit cell apoptosis, and decrease the proportion of cells in $\mathrm{G} 1$ phase (Fig. 6D-6H).

\section{Discussion}

The NF-KB signaling pathway plays an essential role in the initiation and development of human tumors. Accumulating evidence has reported that the NF-KB signaling pathway exhibits abnormal activity in several solid tumors, such as prostate cancer ${ }^{20}$, breast cancer ${ }^{21}$, lung cancer ${ }^{22}$, and $\mathrm{GC}^{23}$. The activity of the canonical NF-KB signaling pathway is mainly based on the inhibitor of IKK, which phosphorylates 
$\mathrm{IKBa}$ and leads to its ubiquitination and degradation ${ }^{18}$. Cumulative studies have indicated that the alterations of $\mathrm{IKBa}$ affect the NF-KB signaling pathway activity, and are subsequently associated with the tumorigenesis of cancers, including lung cancer ${ }^{24}$, breast cancer ${ }^{25}$, pancreatic cancer ${ }^{26}$, and $\mathrm{GC}^{27}$. Therefore, IKBa plays an essential role in the initiation and development of human cancers.

In the present study, we identified that TMEM171 was down-regulated in GC tissues, and GC patients with low expression of TMEM171 had a poorer OS and PFS compared with its high-expression group based on GEO and TCGA datasets. Meanwhile, we found that TMEM171 had a satisfactory sensitivity and specificity of predictive prognosis values for GC patients. Moreover, the bioinformatic analysis showed that TMEM171 was involved in the initiation and development of GC via regulating the $1 \mathrm{KBa} / \mathrm{NF}-\mathrm{KB}$ signaling pathway. Next, we verified that the TMEM171 was downregulated in GC tissues and GC cell lines using our cohort samples. Subsequently, we found that overexpression of TMEM1717 significantly suppressed the proliferation of GC cells, promoted the apoptosis of GC cells, and increased the proportion of cells in the G1 phase, whereas depletion of TMEM171 contributed to an opposite trend in vitro. In vivo, overexpression of TMEM1717 significantly inhibited the growth of nude xenografts. Finally, we found that TMEM171 functioned as an inhibitor for GC proliferation through dephosphorylating $\mathrm{I} B \mathrm{Ba}$ and then inactivating the canonical NF-KB signaling pathway.

As a transmembrane protein, TMEM171 is detected in enterocytes, panteth cells, and cholangiocytes, whereas the function of TMEM171 remains unclear ${ }^{28,29}$. Köttgen $A$ et al. have reported that the TMEM171 is associated with prevalent and painful inflammatory arthritis, which may be a therapeutic target of gout ${ }^{30}$. Xu XL et al. have found that punicalagin (PUN) possesses anti-oxidant and antiinflammatory properties via promoting M2 macrophage polarization and up-regulating TMEM171 31 . Bioinformatic analysis shows that TMEM171 is closely related to the prognosis of lung adenocarcinoma (LUAD), while the underlying mechanism is still unclear ${ }^{32}$. In the present study, we found that the TMEM171 was a promising marker to predict the prognosis of GC patients, and TMEM171 inhibited the proliferation of GC cells via dephosphorylating $\mathrm{IKBa}$ and inactivating canonical NF-KB signaling pathway. Therefore, TMEM171 might be a promising prognostic and therapeutic target of GC in the future.

Accumulating evidence has revealed that the targets and inhibitors of the NF-KB signaling pathway can inhibit the proliferation of cancer cells ${ }^{17,33,34}$. For instance, several phytochemicals including resveratrol ${ }^{35}$, curcumin ${ }^{36}$, and epigallocatechin gallate ${ }^{37}$, suppress the proliferation of cancer cells by blocking the NF-KB signaling pathway. Moreover, miRNAs also play an important role in cancer treatment by inhibiting the NF-KB signaling pathway, such as miR-30 $\mathrm{e}^{38}, \mathrm{miR} 146 \mathrm{a} / \mathrm{b}^{39}, \mathrm{miR}-31^{40}$, and so on. In the present study, our results revealed that TMEM171 targeting the NF-KB signaling pathway might be used as a promising strategy for GC treatment.

\section{Conclusion}


Collectively, we showed that TMEM171 was down-regulated in GC tissues, and its expression was negatively correlated with the prognosis of GC patients. Furthermore, we demonstrated that TMEM171 suppressed the proliferation of GC cells in vitro and in vivo by targeting $\mathrm{IKBa} / \mathrm{NF}-\mathrm{kB}$ signaling pathway. To the best of our knowledge, we, for the first time, identified the role of TMEM171 in the tumorigenesis of GC. Taken together, our current findings provided a novel biomarker for the diagnosis and prognosis of GC.

\section{Abbreviations}

GC, gastric cancer; GEO, Gene Expression Omnibus; TCGA, the Cancer Genome Atlas; IHC, immunohistochemistry; OS, overall survival; LASSO, least absolute shrinkage and selection operator; ROC, receiver operating characteristics; PFS, progression-free survival; DEGs, differentially expressed genes.

\section{Declarations}

\section{Ethics approval and consent to participate}

Study was approved by the Medical Ethics Committee of Shenzhen People's Hospital (LL-KY-2021183). The patients provided their written informed consent in this study.

\section{Authors contributions}

Conception and design: Li-sheng Wang and Jun Yao

Development of methodology: De-feng Li, Yu-ling Bing

Statistical analyses: Qi Tang

Writing manuscript: De-feng Li and Qi Tang

Revising manuscript: Li-sheng Wang and Jun Yao

Study supervision: Li-sheng Wang and Jun Yao

Performed the experiments: De-feng Li, Mei-feng Yang and Quan-zhou Peng

\section{Funds}

This work was supported by Natural Science Foundation of Guangdong Province (No. 2018A0303100024), Three Engineering Training Funds in Shenzhen (No. SYLY201718, No. SYJY201714 and No. SYLY201801), Technical Research and Development Project of Shenzhen (No. JCYJ20150403101028164, No. JCYC20170307100911479, No. JCYJ20190807145617113 and JCYJ20210324113802006), National Natural Science Foundation of China (No. 81800489). 
Acknowledgements

Not applicable.

\section{Availability of data and materials}

The datasets generated during and/or analyzed during the current study are available in the Gene Expression Omnibus (GEO) datasets (http://www.ncbi.nlm.nih.gov/geo/) and TCGA (https://portal.gdc.cancer.gov).

\section{Consent for publication}

Not applicable.

\section{Conflict of Interest}

The authors declare no conflicts of interest.

\section{References}

1. Sung H, Ferlay J, Siegel RL, et al. Global Cancer Statistics 2020: GLOBOCAN Estimates of Incidence and Mortality Worldwide for 36 Cancers in 185 Countries. CA Cancer J Clin 2021;71:209-249.

2. Wang FH, Zhang XT, Li YF, et al. The Chinese Society of Clinical Oncology (CSCO): Clinical guidelines for the diagnosis and treatment of gastric cancer, 2021. Cancer Commun (Lond) 2021;41:747-795.

3. Stahl KA, Olecki EJ, Dixon ME, et al. Gastric Cancer Treatments and Survival Trends in the United States. Curr Oncol 2020;28:138-151.

4. Graham DY. Helicobacter pylori update: gastric cancer, reliable therapy, and possible benefits. Gastroenterology 2015;148:719-31 e3.

5. Friedenreich CM, Ryder-Burbidge C, McNeil J. Physical activity, obesity and sedentary behavior in cancer etiology: epidemiologic evidence and biologic mechanisms. Mol Oncol 2021;15:790-800.

6. Tan P, Yeoh KG. Genetics and Molecular Pathogenesis of Gastric Adenocarcinoma. Gastroenterology 2015;149:1153-1162 e3.

7. Ramezankhani R, Solhi R, Es HA, et al. Novel molecular targets in gastric adenocarcinoma. Pharmacol Ther 2021;220:107714.

8. Yuan L, Xu ZY, Ruan SM, et al. Long non-coding RNAs towards precision medicine in gastric cancer: early diagnosis, treatment, and drug resistance. Mol Cancer 2020;19:96.

9. Li R, Jiang J, Shi H, et al. CircRNA: a rising star in gastric cancer. Cell Mol Life Sci 2020;77:16611680.

10. Kawazoe A, Shitara K. Next-generation sequencing and biomarkers for gastric cancer: what is the future? Ther Adv Med Oncol 2019;11:1758835919848189. 
11. Li D, Yang M, Liao A, et al. Linc00483 as ceRNA regulates proliferation and apoptosis through activating MAPKs in gastric cancer. J Cell Mol Med 2018.

12. Xiao WS, Li DF, Tang YP, et al. Inhibition of epithelialmesenchymal transition in gastric cancer cells by miR711mediated downregulation of CD44 expression. Oncol Rep 2018;40:2844-2853.

13. Li DF, Chang X, Zhao JL, et al. Colonic Epithelial PHLPP2 Deficiency Promotes Colonic Epithelial Pyroptosis by Activating the NF-kappaB Signaling Pathway. Oxid Med Cell Longev 2021;2021:5570731.

14. Yang MF, Long XX, Hu HS, et al. Comprehensive analysis on the expression profile and prognostic values of Synaptotagmins (SYTs) family members and their methylation levels in gastric cancer. Bioengineered 2021;12:3550-3565.

15. Suzuki M, Yokobori T, Gombodorj N, et al. High stromal transforming growth factor beta-induced expression is a novel marker of progression and poor prognosis in gastric cancer. J Surg Oncol 2018;118:966-974.

16. Peng C, Ouyang Y, Lu N, et al. The NF-kappaB Signaling Pathway, the Microbiota, and Gastrointestinal Tumorigenesis: Recent Advances. Front Immunol 2020;11:1387.

17. Liu X, Shao Y, Zhou J, et al. Nuclear Factor kappaB Signaling and Its Related Non-coding RNAs in Cancer Therapy. Mol Ther Nucleic Acids 2020;19:208-217.

18. Espinosa L, Marruecos L. NF-kappaB-Dependent and -Independent (Moonlighting) IkappaBalpha Functions in Differentiation and Cancer. Biomedicines 2021;9.

19. Neumann M, Naumann M. Beyond IkappaBs: alternative regulation of NF-kappaB activity. FASEB J 2007;21:2642-54.

20. Domingo-Domenech J, Mellado B, Ferrer B, et al. Activation of nuclear factor-kappaB in human prostate carcinogenesis and association to biochemical relapse. $\mathrm{Br} J$ Cancer 2005;93:1285-94.

21. Sau A, Lau R, Cabrita MA, et al. Persistent Activation of NF-kappaB in BRCA1-Deficient Mammary Progenitors Drives Aberrant Proliferation and Accumulation of DNA Damage. Cell Stem Cell 2016;19:52-65.

22. Tang X, Liu D, Shishodia S, et al. Nuclear factor-kappaB (NF-kappaB) is frequently expressed in lung cancer and preneoplastic lesions. Cancer 2006;107:2637-46.

23. Sasaki N, Morisaki T, Hashizume K, et al. Nuclear factor-kappaB p65 (RelA) transcription factor is constitutively activated in human gastric carcinoma tissue. Clin Cancer Res 2001;7:4136-42.

24. Ho JY, Lu HY, Cheng HH, et al. UBE2S activates NF-kappaB signaling by binding with IkappaBalpha and promotes metastasis of lung adenocarcinoma cells. Cell Oncol (Dordr) 2021.

25. Yoon HS, Choi SH, Park JH, et al. A Novel Protein-Protein Interaction between RSK3 and IkappaBalpha and a New Binding Inhibitor That Suppresses Breast Cancer Tumorigenesis. Cancers (Basel) 2021;13.

26. Ren X, Chen C, Luo Y, et al. IncRNA-PLACT1 sustains activation of NF-kappaB pathway through a positive feedback loop with IkappaBalpha/E2F1 axis in pancreatic cancer. Mol Cancer 2020;19:35. 
27. Zhang Y, Zhou X, Zhang M, et al. ZBTB20 promotes cell migration and invasion of gastric cancer by inhibiting IkappaBalpha to induce NF-kappaB activation. Artif Cells Nanomed Biotechnol 2019;47:3862-3872.

28. Rolland T, Tasan M, Charloteaux B, et al. A proteome-scale map of the human interactome network. Cell 2014;159:1212-1226.

29. Huttlin EL, Bruckner RJ, Paulo JA, et al. Architecture of the human interactome defines protein communities and disease networks. Nature 2017;545:505-509.

30. Kottgen A, Albrecht E, Teumer A, et al. Genome-wide association analyses identify 18 new loci associated with serum urate concentrations. Nat Genet 2013;45:145-54.

31. Xu X, Guo Y, Zhao J, et al. Punicalagin, a PTP1B inhibitor, induces M2c phenotype polarization via up-regulation of HO-1 in murine macrophages. Free Radic Biol Med 2017;110:408-420.

32. Ren J, Yang Y, Li C, et al. A Novel Prognostic Model of Early-Stage Lung Adenocarcinoma Integrating Methylation and Immune Biomarkers. Front Genet 2020;11:634634.

33. Khan $\mathrm{H}$, Ullah $\mathrm{H}$, Castilho P, et al. Targeting NF-kappaB signaling pathway in cancer by dietary polyphenols. Crit Rev Food Sci Nutr 2020;60:2790-2800.

34. Wu J, Ding J, Yang J, et al. MicroRNA Roles in the Nuclear Factor Kappa B Signaling Pathway in Cancer. Front Immunol 2018;9:546.

35. Ryu J, Ku BM, Lee YK, et al. Resveratrol reduces TNF-alpha-induced U373MG human glioma cell invasion through regulating NF-kappaB activation and uPA/uPAR expression. Anticancer Res 2011;31:4223-30.

36. Ruiz de Porras V, Bystrup S, Martinez-Cardus A, et al. Curcumin mediates oxaliplatin-acquired resistance reversion in colorectal cancer cell lines through modulation of CXC-Chemokine/NFkappaB signalling pathway. Sci Rep 2016;6:24675.

37. Chung SS, Vadgama JV. Curcumin and epigallocatechin gallate inhibit the cancer stem cell phenotype via down-regulation of STAT3-NFkappaB signaling. Anticancer Res 2015;35:39-46.

38. Egan $S M$, Karasik E, Ellis $L$, et al. miR-30 $e^{\star}$ is overexpressed in prostate cancer and promotes NFkappaB-mediated proliferation and tumor growth. Oncotarget 2017;8:67626-67638.

39. Bhaumik D, Scott GK, Schokrpur S, et al. Expression of microRNA-146 suppresses NF-kappaB activity with reduction of metastatic potential in breast cancer cells. Oncogene 2008;27:5643-7.

40. Uribesalgo I, Ballare C, Di Croce L. Polycomb regulates NF-kappaB signaling in cancer through miRNA. Cancer Cell 2012;21:5-7.

\section{Figures}



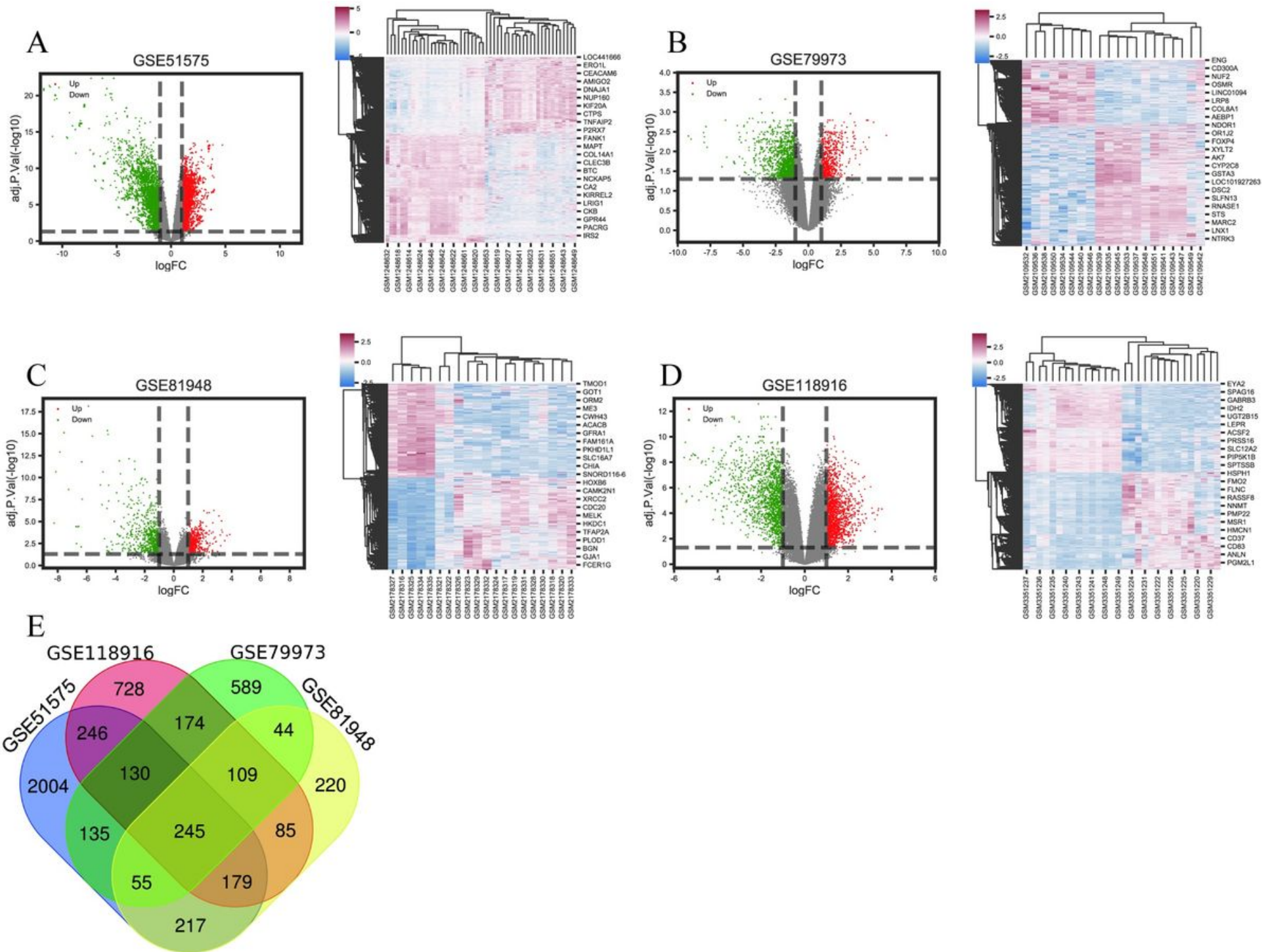

Figure 1

The DEGs between GC samples and normal tissues from GEO datasets. A, The DEGs in GSE51575; B, The DEGs in GSE79973; C, The DEGs in GSE81948; D, The DEGs in GSE118916; E, Overlapping DEGs in GSE51575, GSE79973, GSE81948, and GSE118916. 


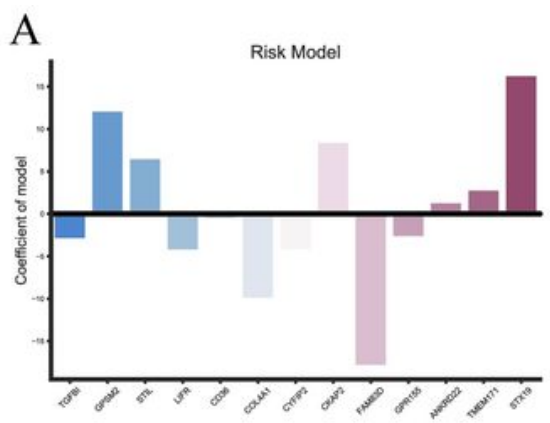

B
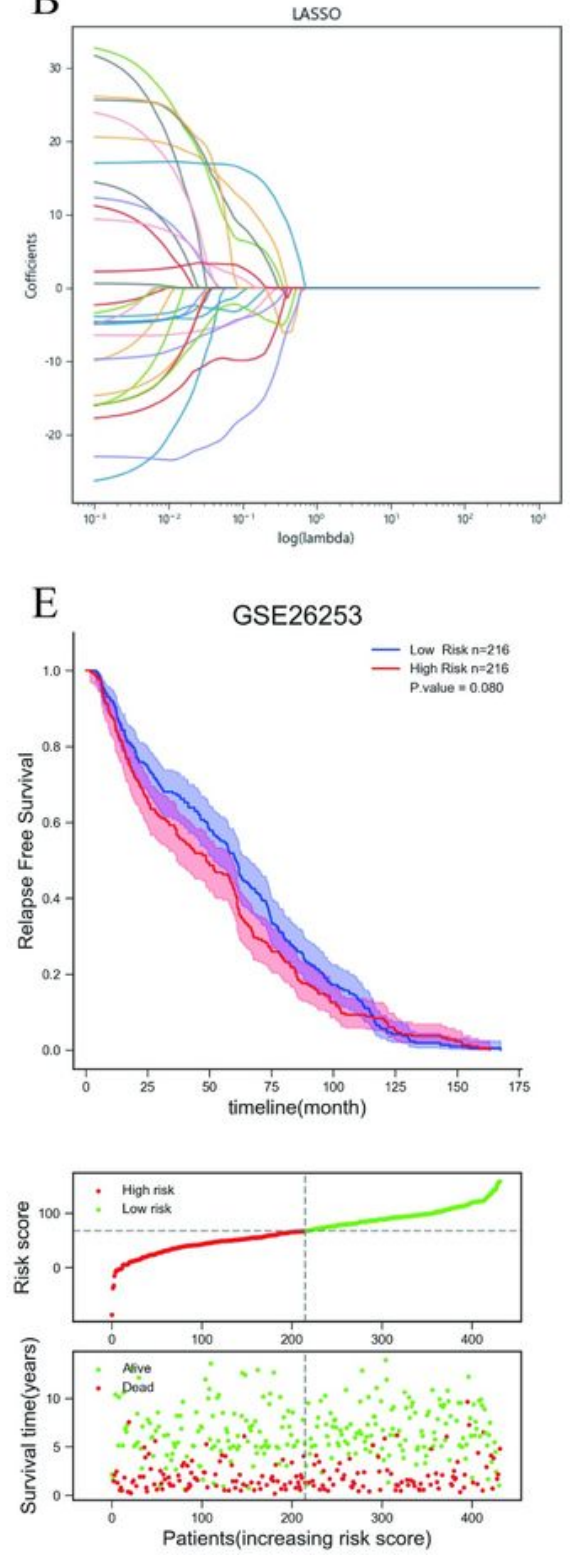
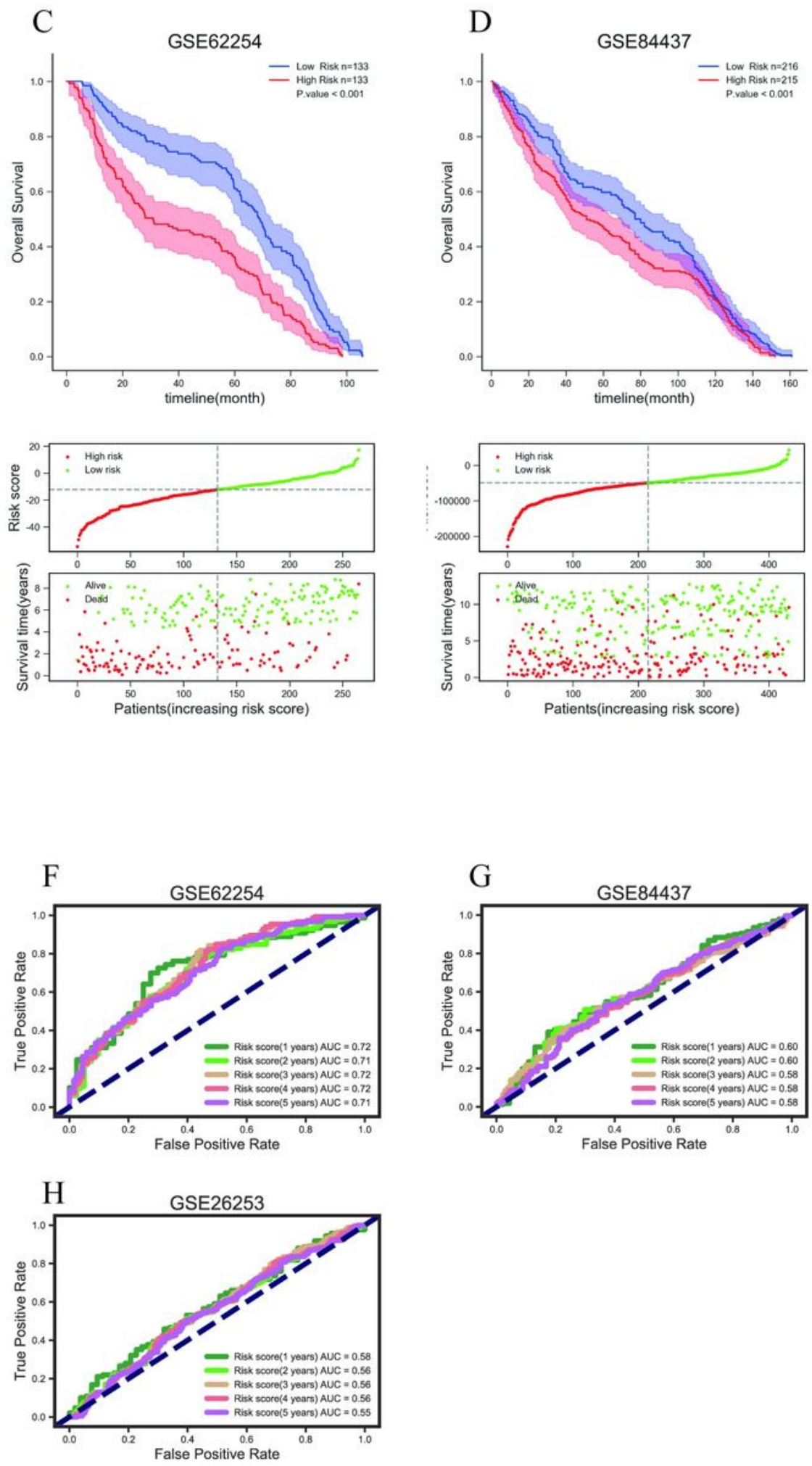

Figure 2

A risk scoring model construction based on the overlapping DEGs. A, B, LASSO regression analysis for screening prognosis-related genes and constructing a risk scoring model; C, D, E, Survival analysis based on the risk scoring model in GSE62254, GSE84437, and GSE26253, survival curve for low-risk and highrisk groups (upper), risk score distribution (middle), and survival status (bottom); F, G, H, The ROC curves for predicting OS based on risk score in GSE62254, GSE84437, and GSE26253. 


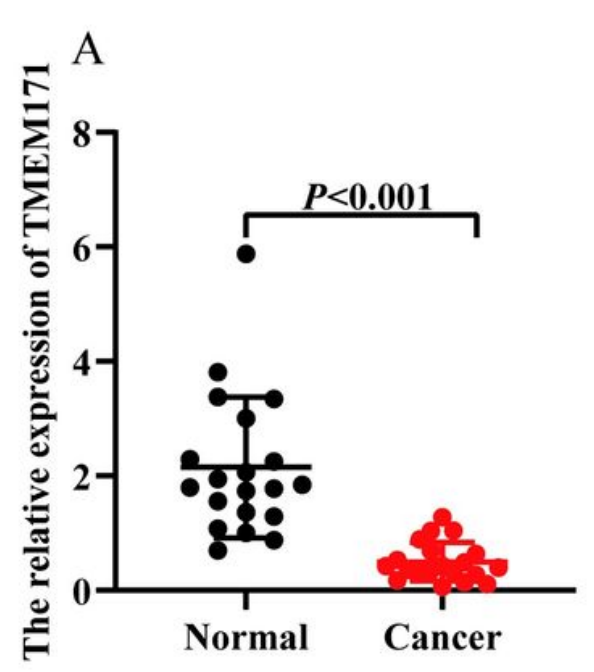

B
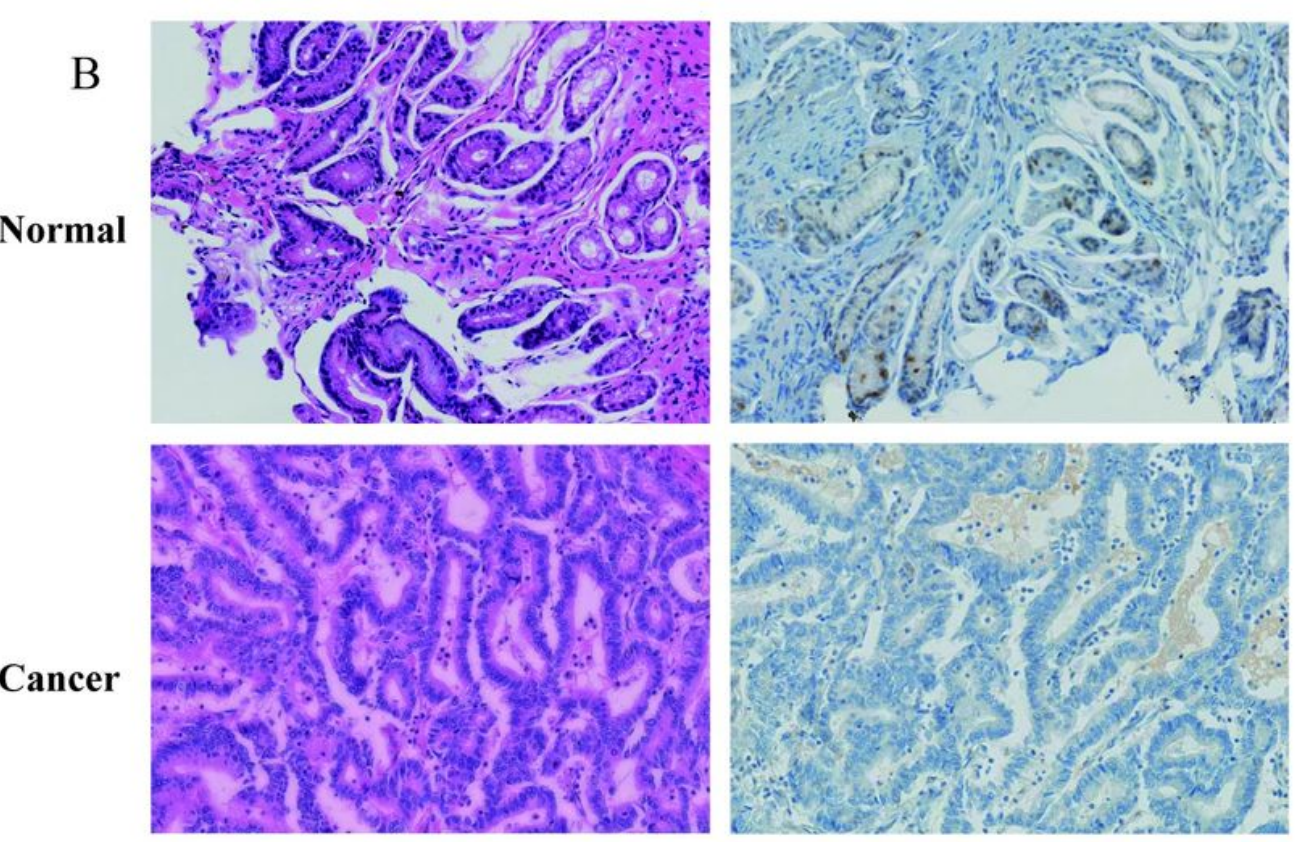

HE

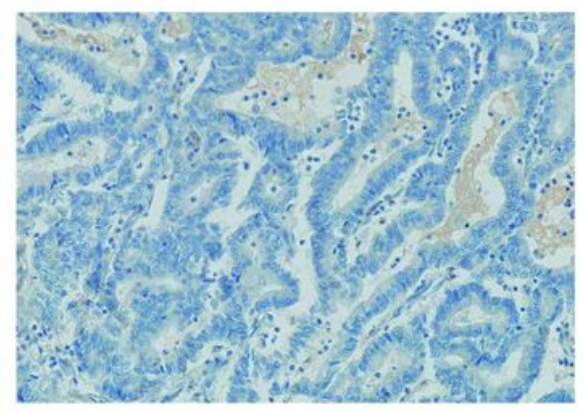

IHC (TMEM171)

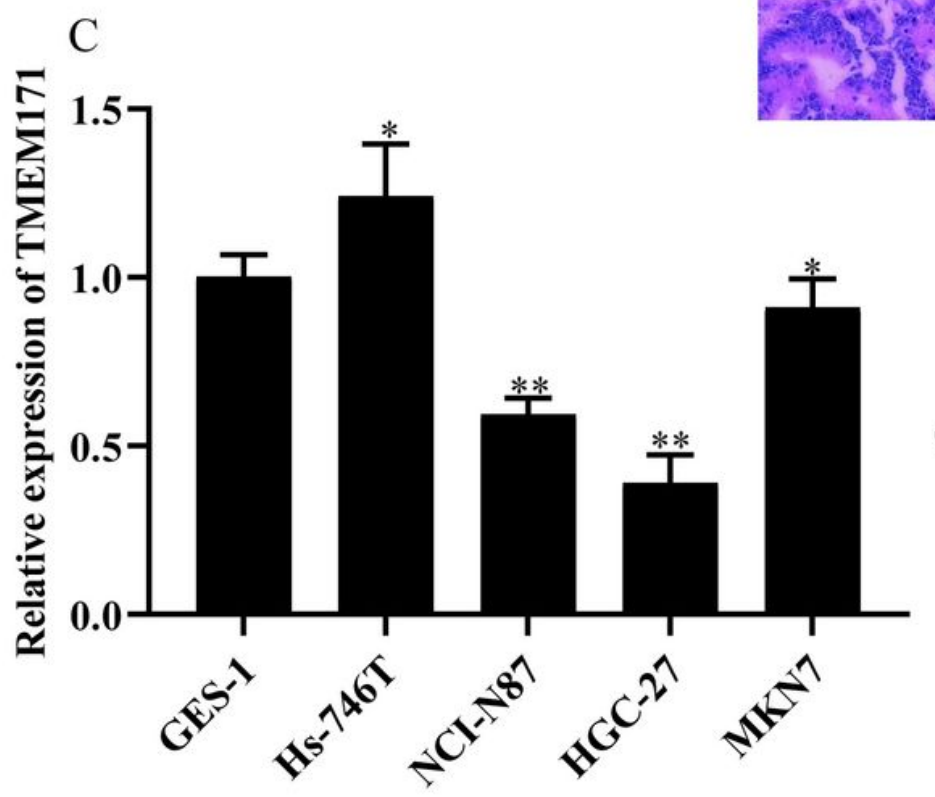

$\mathrm{D}$

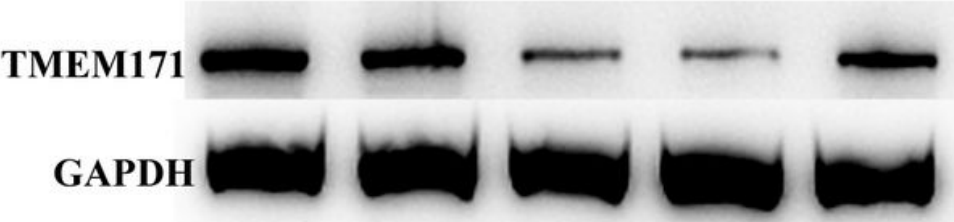

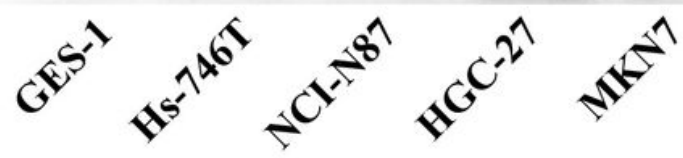

Figure 3

The expression of TMEM171 in GC. A, The expression of TMEM171 at the mRNA level was significantly decreased in GC samples; $B$, The expression of TMEM171 at the protein level was significantly downregulated in GC samples (200x); C, D, The highest expression of TMEM171 was found in Hs-746T cells, whereas its lowest expression was detected in HGC-27 cells. 

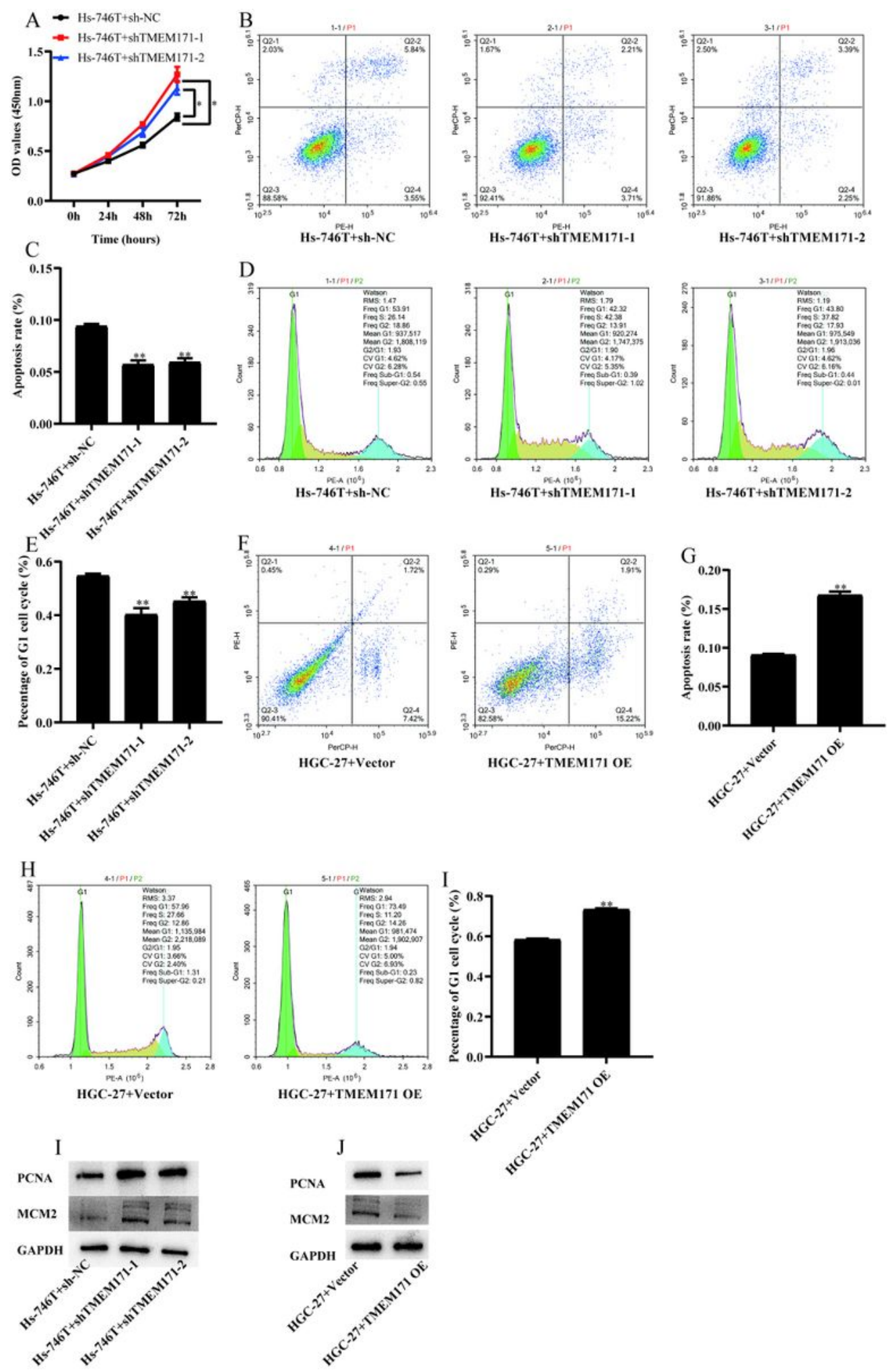

\section{Figure 4}

TMEM171 inhibits the proliferation of GC cells in vitro. A, Depletion of TMEM171 promoted the proliferation of Hs-746T cells; B, C, Depletion of TMEM171 inhibited the apoptosis of Hs-746T cells; D, E, Depletion of TMEM171 decreased the proportion of Hs-746T cells in the G1 phase; F, Overexpression of TMEM171 suppressed the proliferation of Hs-746T cells; G, H, Overexpression of TMEM171 promoted the apoptosis of Hs-746T cells; I, J, Overexpression of TMEM171 increased the proportion of Hs-746T cells in 

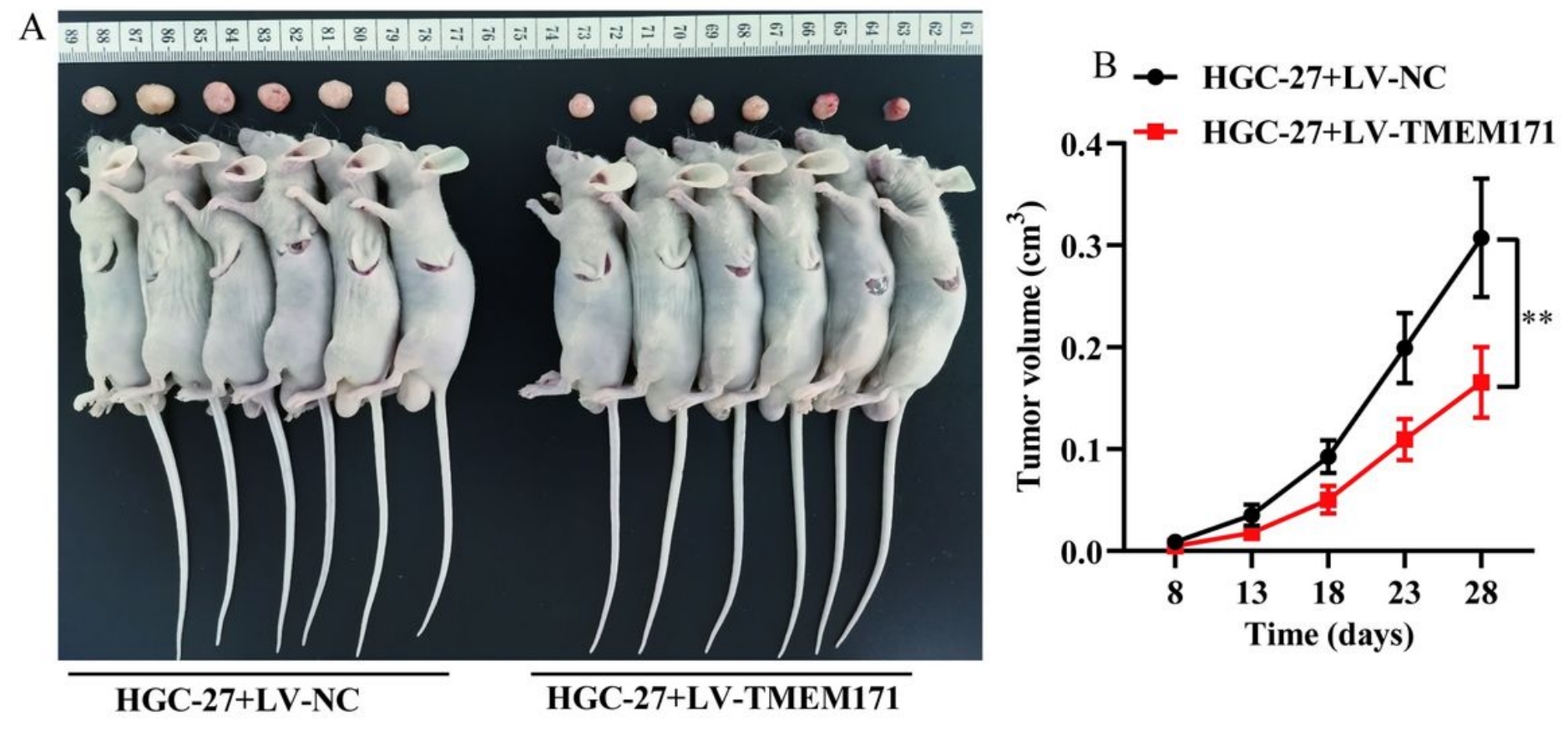

C
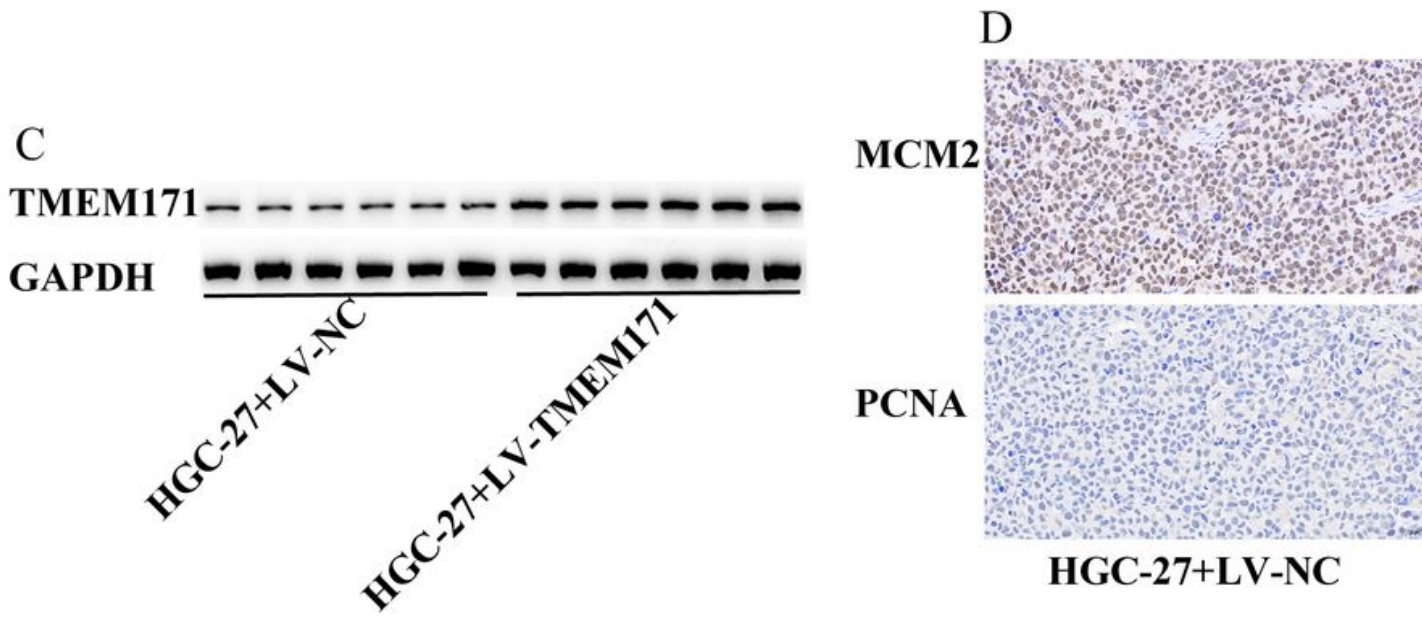

HGC-27+LV-NC

HGC-27+LV-TMEM171

Figure 5

TMEM171 inhibits the proliferation of GC in vivo. A, B, Overexpression of TMEM171 inhibited the growth of xenografts; C, The expression of TMEM171 at the protein level was significantly increased in the TMEM171 overexpression group; D, Overexpression of TMEM171 decreased the expressions of MCM2 and PCNA at the protein level in xenografts. 

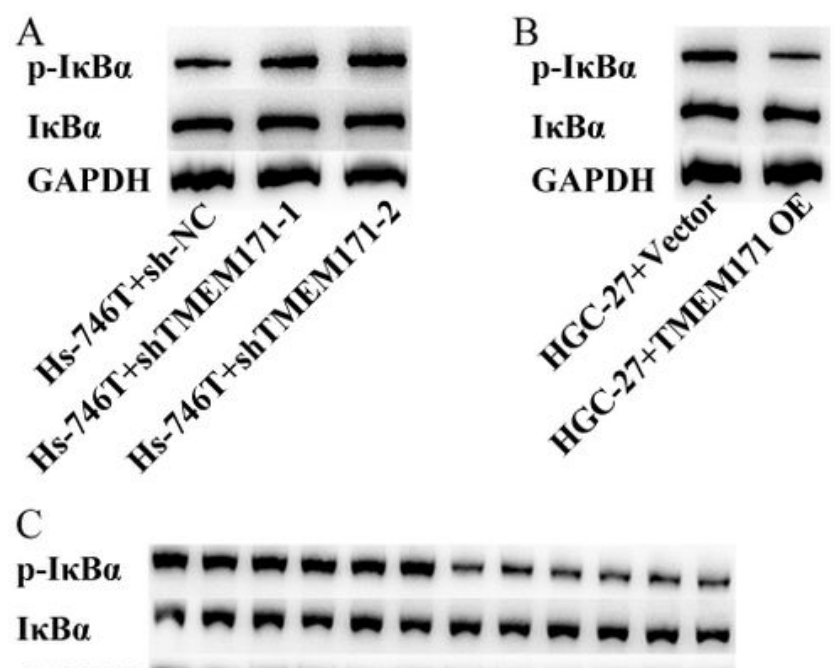

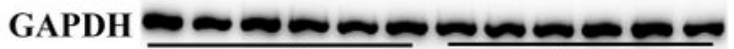

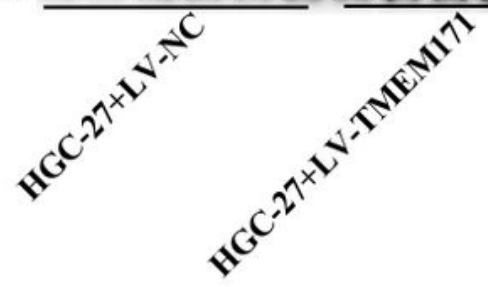

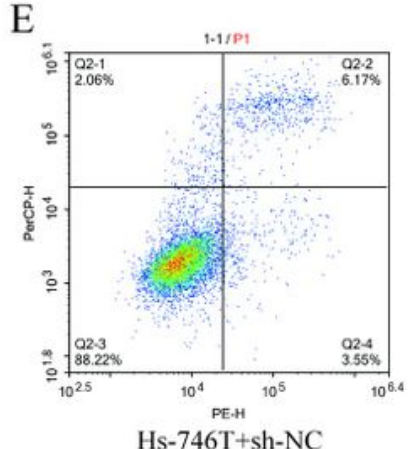

Hs-746T + sh-NC

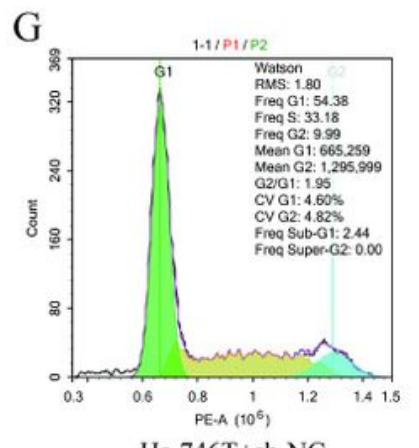

Hs-746T + sh-NC

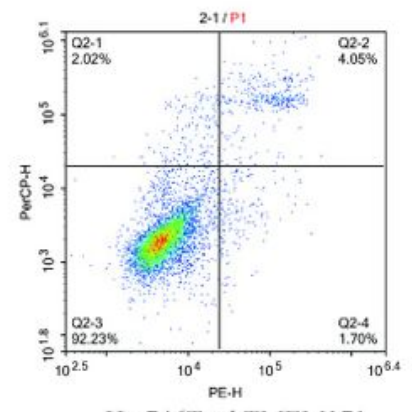

Hs-746T+shTMEM171

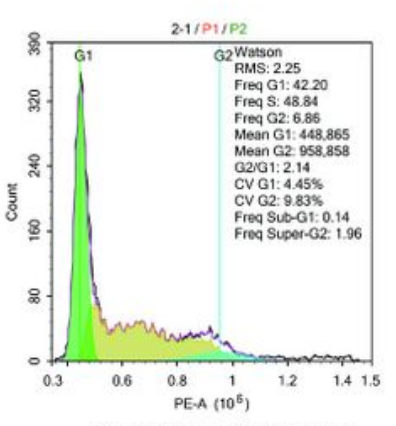

Hs-746T+shTMEM171

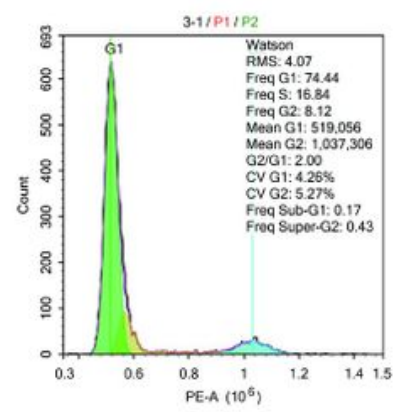

Hs-746T + sh-NC+PDTC
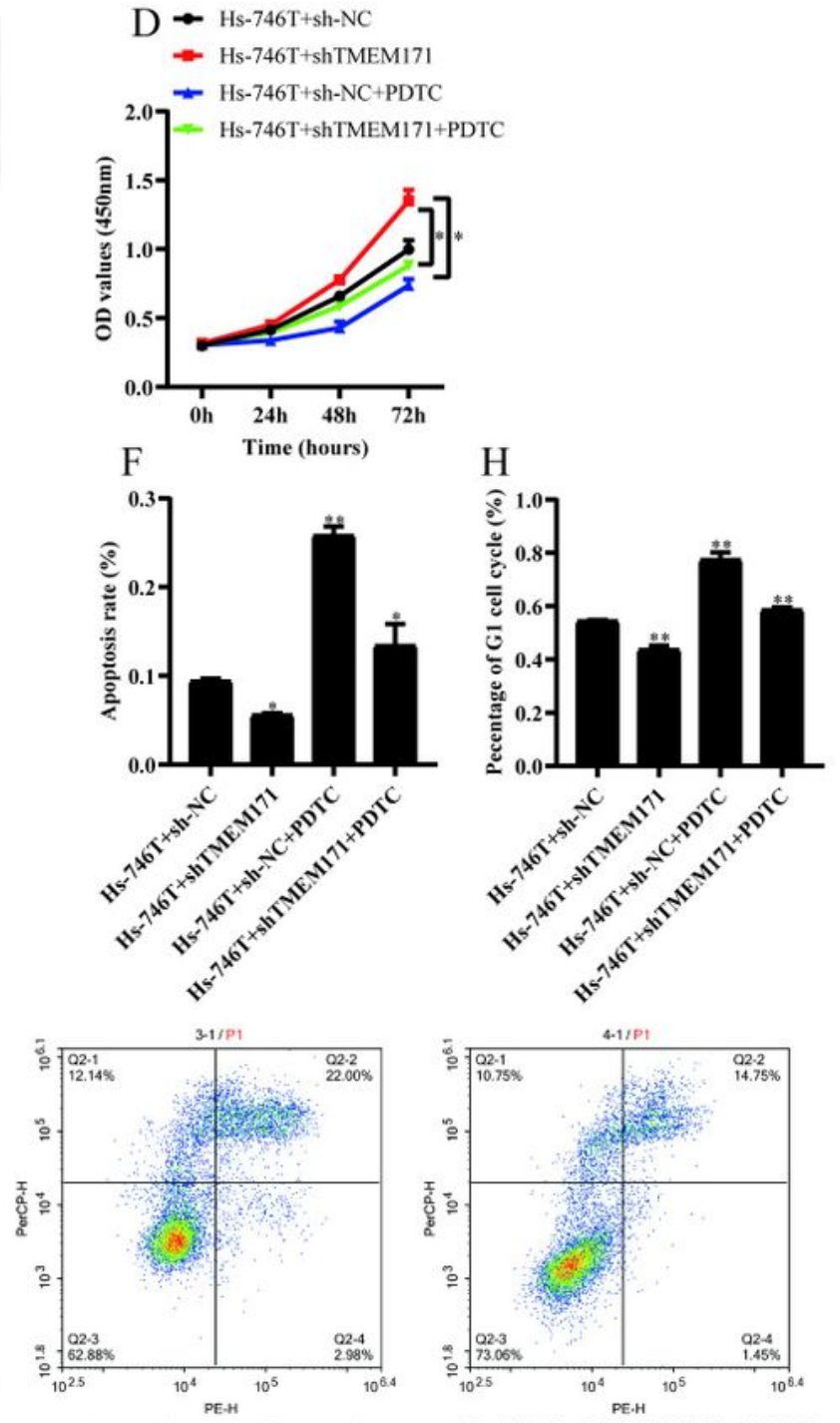

Hs-746T+ shTMEM171+PDTC

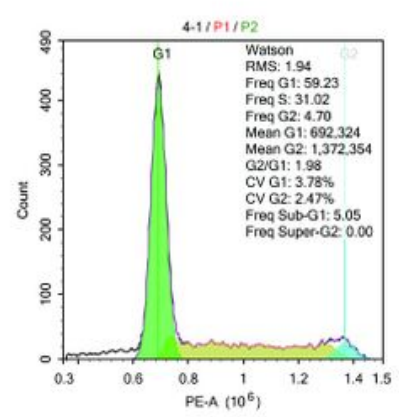

Hs-746T+ shTMEM171+PDTC

Figure 6

TMEM171 dephosphorylates IKBa and inactivates the NF-KB signaling pathway. $A, B$, The overexpression or depletion of TMEM171 exhibited no significant effect on the expression of IKBa at the protein level, whereas overexpression of TMEM171 reduced the expression of p-IKBa, and depletion of TMEM171 resulted in increased expression of $\mathrm{p}-\mathrm{I} \mathrm{KBa}$ in vitro; $\mathrm{C}$, Overexpression of TMEM171 had no significant effect on the expression of IKBa at the protein level, while overexpression of TMEM171 decreased the 
expression of $\mathrm{p}-\mathrm{I} \mathrm{KBa}$ in vivo; $\mathrm{D}, \mathrm{E}, \mathrm{F}, \mathrm{G}, \mathrm{H}, \mathrm{PDTC}$, an NF-KB signaling pathway inhibitor, could rescue TMEM171 depletion, promote cell proliferation, inhibit cell apoptosis, and decrease the proportion of cells in the $\mathrm{G} 1$ phase in vitro.

\section{Supplementary Files}

This is a list of supplementary files associated with this preprint. Click to download.

- supplement.docx 